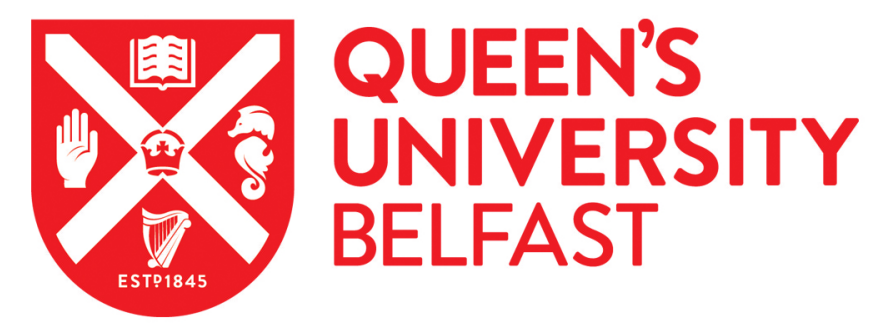

\title{
Family perceptions of care at the end of life in UK nursing care homes
}

Kinley, J., Hockley, J., Stone, L., \& Brazil, K. (2018). Family perceptions of care at the end of life in UK nursing care homes. Journal of Research in Nursing. https://doi.org/10.1177/1744987117753276

Published in:

Journal of Research in Nursing

Document Version:

Peer reviewed version

Queen's University Belfast - Research Portal:

Link to publication record in Queen's University Belfast Research Portal

Publisher rights

(c) 2018 The Authors. This work is made available online in accordance with the publisher's policies. Please refer to any applicable terms of use of the publisher.

\section{General rights}

Copyright for the publications made accessible via the Queen's University Belfast Research Portal is retained by the author(s) and / or other copyright owners and it is a condition of accessing these publications that users recognise and abide by the legal requirements associated with these rights.

Take down policy

The Research Portal is Queen's institutional repository that provides access to Queen's research output. Every effort has been made to ensure that content in the Research Portal does not infringe any person's rights, or applicable UK laws. If you discover content in the Research Portal that you believe breaches copyright or violates any law, please contact openaccess@qub.ac.uk. 
Family perceptions of care at the end-of-life in UK nursing care homes

\section{Authors:}

Julie Kinley, Jo Hockley, Louisa Stone, and Kevin Brazil

\section{Corresponding author:}

Julie Kinley

Care Home Project Team

St Christopher's Hospice

London SE26 6DZ, UK.

j.kinley@stchristophers.org.uk

02087684727

\section{Other authors:}

Dr Jo Hockley

University of Edinburgh

Edinburgh, Scotland, UK

Louisa Stone

Care Home Project Team

St Christopher's Hospice

London, UK.

Professor K Brazil

University of Belfast

Belfast, Northern Ireland, UK

FUNDING: This research study was funded by The Wives Fellowship and St

Christopher's Hospice.

CONFLICT OF INTEREST: The authors declare that there is no conflict of interest. 


\begin{abstract}
Background: Over a fifth of the population of developed countries die in care homes. Within the UK a number of national end-of-life care documents have been introduced to guide such care provision. Whilst studies are emerging on the outcomes of care in the last few weeks of life, few report on the experience as perceived by the family members. As part of a wider study to improve the delivery of end-of-life care, bereaved relatives of residents who had died at the care home/hospital were sent the Family Perception of Care Scale in order to address this.
\end{abstract}

Aim: To evaluate the experience of care provision in the last month of life as perceived by bereaved relatives.

Design: A cross-sectional design

Setting: Nursing care homes in south-east England.

Participants: Bereaved relatives of residents within 37 nursing care homes implementing the Gold Standards Framework in Care Homes programme.

Methods: The Family Perception of Care Scale questionnaire was posted to bereaved relatives, from 37 nursing care homes, 3-6 months following the resident's death. The questionnaires were posted over a 15month period from October 2009 - December 2010. Results: A total of 869 questionnaires were posted with a $42 \%$ response rate. A global question within the FPC scale looking at the overall satisfaction with the quality of end of life care (Q24) indicated that bereaved relatives were satisfied with the care provided. However, qualitative responses from family members highlight underlying issues with: communication and relationships in care homes; poor professional teamwork; aspects of dying; and, spiritual care. 
Conclusions: The qualitative data from these questionnaires provides an important insight into care provision at the end-of-life within these care homes.

Key words: Nursing homes; end-of-life; quality of care; bereaved families; long-term care 


\section{INTRODUCTION:}

Quality care at the end-of-life in care homes has emerged as a critical health policy issue internationally (DH 2013; Fisher \& Ross 2000; Swagerty 2017). Despite increased use of hospice services and developments in end-of-life care in nursing care homes (NHs) in the USA, Canada and UK, the quality of care given to residents in the last month of life is not widely researched (Li et al 2013; Hockley et al 2010). In NHs in the UK, 56\% residents die within a year of admission and so care provision at the end-of-life is an important consideration (Kinley et al 2013).

Older people in care homes comprise a diverse population with a burden of chronic and terminal conditions including high rates of dementia (Alzheimers Society 2015). Consequently there are a number of challenges to the provision of good end-of-life care. These include: identifying prognosis (Wilkinson \& Lynn 2005; Lunney et al 2003); pain and symptom management (Husebo et al 2011; Kaasalainen 2012); a lack of appropriate assessment and drug prescribing (Hanson \& Henderson 2000); and, advance care planning which is often not attempted or completed comprehensively in NHs (Froggatt et al 2009; Stone et al 2013). As a result of such factors, unnecessary hospitalisation is common (Harrison et al 2016) . Furthermore, educational gaps in the training of care home staff coupled with communication problems between health care providers, family members, and residents represent major challenges to providing quality end-of-life care (Oliver et al 2004; Brazil et al 2006; Zheng et al 2010). 
User satisfaction has become a key attribute to measure the quality of care provided (Jeon et al 2012; Healthwatch 2017). For researchers, policy makers and commissioners assessing 'satisfaction with care' may be used as an evaluative outcome. For care home directors, regular measurement of user satisfaction can assist organisations in meeting regulatory requirements. User satisfaction information is becoming the basis for comparing service delivery and improvement (Brazil 2009). However, satisfaction with care is rarely undertaken to measure quality in end-of-life care in NHs. A systematic review looking at validated tools to assess satisfaction with end-of-life care in care homes

recommended the Family Perception of Care Scale (FPCS) within this care setting (Parker \& Hodgkinson 2010). The FPCS, developed in Canada, contains 25 'likert' type questions relating to four domains: resident care, family support, communication and rooming [physical environment] (Vohra et al 2006).

This article reports data from bereaved relatives' satisfaction with end-of-life care through their completion of the FPCS. Data are taken from a wider study evaluating different ways of facilitating a UK practice development programme in end-of-life care in NHs (Kinley et al 2013; Kinley et al 2014; Hockley et al 2013). In the UK, NHs are care homes that have on-site nurses working alongside care workers but they rely on external medical support.

\section{METHODS:}

A cross-sectional postal questionnaire survey was undertaken to evaluate bereaved families perception of theprovision of end-of-life care across $37 \mathrm{NCHs}$ in south-east 
England. The next-of-kin was sent the FPCS questionnaire 3-6 months after the death as part of a wider study (Kinley et al 2014). Individuals were excluded if: the relative had physical/mental health problems preventing completion of the questionnaire; the next-ofkin had already had a bereavement of another relative within the NH during the study; or, the next-of-kin would be unable to complete the questionnaire in English. They were sent out over a 15month period (October 2009 - December 2010).

The questionnaire and supporting documentation (letter signed by $\mathrm{NH}$ manager, information sheet, consent form, 'decision not to participate' form, and details of a social worker, external to the research team, who could give support if necessary) were sent with a stamped addressed envelope to the next-of-kin. Instructions were given to return either the questionnaire or the 'decision not to participate' form to the research team; when either were not returned within four weeks, one reminder letter was sent from the NH manager. A 'thank you' card was sent to those returning completed questionnaires.

Ethics approval was granted by the Joint UCL/UCLH Committees on the Ethics of Human Research in 2009. REC reference number: 09/H0715/74. The larger study was registered on the ISRCTN Register (Trial Number: ISRCTN76029577).

An adapted version of the FPCS was used in this study. The original questionnaire consists of 25-items where bereaved relatives indicate their agreement as rated on a seven-point Likert scale (Vohra et al 2006). Also, they identify three of the items as priorities for providing quality end-of-life care. An opportunity is given to include written 
comments. For the purposes of this study, some changes to the original FPCS were made to fit the UK context, namely:

- minor changes to the phrasing of seven questions

- removal of Q19. 'My family member was placed on an appropriate floor/unit'. This question was removed, with permission from those who validated the original form, as it was non- applicable to this study which only took place in nursing care homes where residents were not moved.

- FPCS questionnaire was posted to all bereaved relatives, irrespective of the residents place of death,

- a 'not applicable' option was also provided.

- total possible score ranged from 24 (negative assessment) to 168 (highly positive assessment).

- bereaved relatives were also asked to identify: place of death; relationship to the deceased resident; and, their age.

Data analyses of the quantitative data were undertaken using IBM SPSS version 21. Data are presented as frequencies and mean (SD). Overall respondent satisfaction was calculated by adding the respondent responses to all FPCS questions. Thematic analysis was used to analyse respondents' hand-written comments. Initial analyses involved a research analyst reviewing the responses, and identifying themes. Two further researchers reviewed the themes where differences were resolved through a process of clarifying and redefining themes. 


\section{RESULTS:}

\section{Quantitative:}

The FPCS questionnaire was sent to 869 bereaved relatives from 37 NHs generating a response rate of $42 \%(366 / 869)$. Most respondents were sons or daughters of the deceased resident $60.0 \%$ (see Table 1 ).

[Table 1 - about here]

Seventy-seven percent of residents $(277 / 366)$ died in the NH. The average number of beds in the participating NHs was 59 beds (range 22-160). Seventy per cent of NHs were 'for profit' organizations, with 54\% being part of large cooperatives (see Table 2).

[Table 2 - about here]

All analyses on the FPCS were collapsed across NHs. Overall, bereaved relatives were satisfied with the quality of end-of-life care provided revealing an overall mean score on the adapted FPCS of 135 (SD 30.17) from a possible total score of 168 (see Table 3).

[Table 3 - about here]

Whilst all questions were positively rated by bereaved relatives (mildly to strongly agree), the lowest ranked questions (mildly agree) clustered around communication between staff and family on what to expect at the end-of-life. Questions in this section of the FPCS included: 'staff described what to expect as my family member came closer to death'; 'staff informed me about care options during my family member's last days'; 'staff involved me in the planning of care'; 'staff asked about and met important rites and rituals of my family member/us'. 
The other area of care where bereaved relatives were only mildly satisfied was with the availability of spiritual support.

Bereaved relatives identified three items in the FPCS they reported most important for excellent end-of-life care (Table 4). 'Staff treat their family member with dignity' was the highest ranked by bereaved relatives; followed by 'effective pain control' and 'being sensitive to the needs of their family member' including 'comfort care' and 'effective symptom management'

[Table 4 - about here]

\section{Qualitative:}

Out of the 366 returned FPCS, 30\% (110/366) next-of-kin had written comments on the FPCS. A number of respondents specifically stated they found filling in the FPCS helpful. Four themes were identified from the free-style comments:

\section{Communication and relationships in the care home:}

Good communication both with the relative and their family member was seen as paramount to good end-of-life care. Families wanted a friendly human face - people that were 'warm, caring and genuine'.

'It is very important that the relatives and home have a good relationship. I had absolute confidence in the staff at [NH].' [R.18]

Some relatives remarked that there was too much rushing around at times for the resident to be respected and listened to; as one person put it they wanted 'more tender loving care'. 
In some NHs, it was relatives who shaped the care their family member received and appeared a constant struggle for relatives to get the appropriate care they believed their family member required.

'Each time it was up to the family to bring these factors to the forefront so as to be dealt with accordingly - but should have already been in place automatically.' [R.56] Some families referred to preferences and wishes about end-of-life care. If there was no plan and symptoms suddenly became a problem in the last days of life it was likely admission to hospital would occur. However, having an advance care plan documented did not necessarily guarantee wishes and preferences were carried through.

'I was very sad to hear that the nursing staff had discussed with my father that he should stay with them to die. He then asked me when he was going home and expressed a wish to die in his own home. I felt desperately sad and inadequate as I had not been consulted and it was then too late to implement his dying wish. This I will never forget.' [R.32]

Most times when the family member and relatives were both involved, it worked well. '... when the hospital said Mum had to go into care I was worried. I live in Yorkshire she in [SE England] and didn't know what to expect. [The NH] was wonderful. Mum had the best care possible and were so friendly and helped me no end...didn't mind if I stayed all night near the end and even though Mum was not there long before she died; they felt like extended family.' [R.31]

Given the challenges of providing high quality end-of-life care in care homes, as identified earlier, this was provided where care home staff had acknowledged its 
importance alongside the need to form good relationships with the families of residents (despite some being many miles away):

'End-of-life care for my mother was outstanding. What stood out were: kind, sensitive staff; full 24-hr access and privacy; easy communication at all stages; totally professional at all times; outstanding care.’ [R.21]

\section{Professional Teamwork}

Where staff worked well together with the inclusion of the GP, the care home was praised. Unfortunately, this was not always the case. Examples were given of poor professionalism and teamwork. For example, relatives reported that they had wanted assurance that their family member had seen a doctor. They were supplied with correct, but misleading, information.

'I asked the nursing staff if a doctor would be attending and they confirmed an emergency doctor had been called. I later learnt that he did not attend but merely held a telephone conversation with someone on the nursing staff.' [R.26]

Again, whilst eight families specifically mentioned the support they valued receiving from specialist palliative care teams in another case advice from specialist palliative care was not carried through:

'We had stand-up arguments with some of them as she writhed in pain in her bed! Despite written advice from [the hospice] in front of them, I had to get them to call [the hospice] .....' 
It was important for relatives to see good professional teamwork continue throughout the whole process - even after the death. If humane care didn't continue to after the resident had died, then good care given prior to the death was in danger of being discounted.

'I was dismayed that within one and a half hours of his death, my father had become "the body" to be removed immediately... This lack of sensitivity nearly obviated my appreciation of the compassion which had been exhibited to my father and to me during the previous few days.' [R.47]

\section{Aspects of dying:}

Sixteen relatives specifically talked about the good death that had occurred in the NH:

'As it was she died in my arms at the NH. It was a 'good death'..... In the last week I was there for hours at a time - and welcomed by the staff.' [R.43]

Many family members mentioned how they wanted to be there at the death - some were disappointed not to be called in time. However, it was clear from eleven relatives' comments that staff in some of the care homes either didn't really understand the process of dying or felt unable to speak about it to relatives. This was still the case even when the relative knew their family member was dying:

'They seemed reluctant to accept that she was dying preferring rather to lecture me on the importance of getting her out of bed "to prevent contractures" [R.121]

Pain and breathing difficulties were the main symptoms that relatives commented on during the process of dying. Breathing difficulties were frightening and in many instances appeared to come on suddenly often precipitating hospital admission. If anticipatory medication for symptoms during the last days of life were available then often residents 
remained in the $\mathrm{NH}$ and died peacefully. However, one relative spoke passionately that medication at the end-of-life had made their family member confused and distressed.

Twenty-three relatives commented that their family member died 'suddenly'.

'My mother-in-law was nearly 100 years old when she died. This was quick and without pain; she had her hair done in the morning and looked 'lovely'. [R.35]

Those residents whose disease trajectory deteriorated as a result of an 'acute' episode (falling and fracturing a bone; a stroke) were more likely to be admitted to hospital. This was the case for twenty-four relatives. In these instances a few respondents used the FPCS to comment negatively on the hospital care:

'My mother suffered a massive stroke and although she was admitted into the hospital nearly two hours before I could get there, I had to watch her die on a gurney in a cramped dingy A\&E cubicle. It was horrible’. [R.22]

Twenty-one relatives (19\%) specifically mentioned that there were not enough staff and sadly one incident was related to dying:

'My wife passed away while I was with her but it took some time to find a member of staff to tell her she had gone.' [R.18]

These shortages were often reported in relation to care provision at nights or weekends but one person talked about a lack of nurses generally in a $\mathrm{NH}$.

\section{Spiritual care:}

Spiritual care was important for many relatives, many of whom wanted more input especially when someone was dying. One person in particular mentioned how the priest had visited the family member in the $\mathrm{NH}$ for well over five years. Most relatives appeared to organise spiritual care themselves. 
'Our Methodist minister came to see my husband. There was no clergyman who visited [the home] regularly.' [R.28]

Many relatives spoke about the respectfulness with which their family member and indeed themselves were treated following the death.

'Tea and biscuits were there if needed. She was respectfully treated when doctors/ funeral people came.' [R.36]

Staff attending the funeral was appreciated by relatives. This was most often seen when a rapport had been built up over time with the staff. However, when a staff member had said they would be attending the funeral, but for some reason didn't there was disappointment. One respondent who was a nurse specialist in palliative care mentioned that she was very impressed by the care that other residents got following the death of her mother and the openness when a resident died.

\section{DISCUSSION:}

When evaluating service delivery and provision of care, few studies account for both the outcome and the experience of care delivery (quantitative and qualitative data) (Seers et al 2012;+a more recent ref). In this study, the quantitative analysis reports a largely favourable experience concerning the end-of-life care provision for their family member in NHs. However, the overall qualitative analysis, while supporting the positive quantitative data, does reveal nuances of underlying problems. Like all qualitative studies, the data highlights important detail; without such data it is difficult to know what to target when improving care. 
Considering we were asking bereaved relatives to complete the questionnaire, there was a good response rate (42\%). A strength of the FPCS is that it was specifically validated for use within a care home setting (Vohra et al 2006). This short 5-page document means it is potentially useful as an audit tool (Levy et al 2016). Encouraging audit in this care sector as a means of improving the care they are delivering may be very appropriate (Davies et al 2014; Hockley and Kinley 2016). With $80 \%$ of residents in a care home having a severe memory problem or dementia the validated FPCS captures the experience of care where residents cannot provide a personal account of their experience. Learning from and improving practice will be enabled if further validation of FPCS for care homes across countries occurs. In addition commissioners of care for frail older people might also be interested in such a tool.

One of the main concerns of relatives in this study was the lack of medical support. All study NHs were enrolled in a programme (www.goldstandardsframework.org.uk) to improve end-of-life care. One aim of this programme is the building of relationships with external healthcare professionals (GSF 2009). In spite of this, relatives found medical input lacking. Medical input to UK NHs is intermittent and insufficient (Kinley et al 2014; Handley et al 2014; Jacobs 2003). This has been the case since the closure of NHS geriatric wards in the 1990s. Funding was given to social services to provide care for frail older people in their own homes and care homes, with little consideration of medical input (BJS 2011). The majority of older people now admitted to NHs are frail with multimorbidities and die within a year of admission (Kinley et al 2013). This may suggest the 
need for a new model of medical provision. Alternative medical models for UK NHs do exist: doctors employed by the NH (Baar 2002); retainer fees for GPs to attend the NH more regularly (Hockley et al 2015; Handley et al 2014); and even a dedicated primary care team for NHs in the city of Glasgow which sadly has now been disbanded because of equity issues (Hannah et al 2005). Although 43\% NHs in our study had a dedicated GP practice, the qualitative findings reveal that some families were critical about the quality of service provided. A report from the British Geriatric Society recommends a combination of 'enhanced primary medical and nursing care with dedicated input from departments of old age medicine, mental health services, and other specialisms such as palliative care and rehabilitation medicine according to local needs'(BGS 2011:4). In the USA there has been a tradition for the development of teaching nursing homes being aligned to local universities to achieve high quality care (Mezey et al 2009; add your reference). Nationally further improvments in medical provision may occur through the recommendations emerging through the six care home vanguard sites (NHS England 2016); the NHS five year forward plan (NHS England 2017) and their accociated Sustainability and Transformation plans https://www.england.nhs.uk/stps/ and innovative suggestions posed e.g. increased use of information technology. The use of technology may offer more that greter access to medical support it may also provide a means of gaining user feedback about the experience of care.

Re-thinking different models of care provision is not just about medical care, spiritual care was important for residents in their last month of life in the $\mathrm{NH}$ as reported in the FPCS. Whereas twenty years ago chaplain support would have been part of the provision 
of end-of-life care in geriatric hospitals, this is not routinely provided in NHs. A new concept, Parish Nursing, currently emerging in the USA and the UK might offer a solution (Wordsworth 2014). Even in cancer care the emergence of validated tools to Identif ??measure and improve such care is just emerging (Vivat et al 2017). Engaging care homes with those developing such tools would be one way to better ensure the care home population also is included.

Relationships between care staff, nurses, management in NHs, GPs, the resident and family were extremely important to those responding to the FPCS. There is considerable discussion about person-centred care in NHs which has tended to be interpreted as resident focused care. Establishing instead a culture of relationship-based care enhances communication across all involved and has been shown to transform the culture of care (Koloroutis 2004) + newer ref.

Recognising dying is fundamental to managing the last day/s or week/s of life (Ellershaw \& Wilkinson 2003). In the past, diagnosing dying has been the role of the doctor. Where NH staff were inadequately supported by GPs, confidence in acknowledging dying was lacking. This situation is compounded by the fact that the dying trajectory of very frail older people is often difficult to identify (Murray et al 2005). Given such challenges it could be argued that most of the NHs, in this study, were perceived as giving high quality end-of-life care by those who returned the FPC questionnaires. Since this study the ability to do this well has been further enabled with the publication in the UK of the nationally recommended five prioroities of care for the dying person documnent (add ref). An early 
audit reports further positive experience when GPs and nursing care home staff work together to provide care to residents in their last days of life (Coleman et al 2017).

A limitation in this study was that the FPCS was adapted to suit the UK context (as previously explained Q19 was removed). This meant that a validated tool within Canada was not a validated tool for this study but was reported as the most appropriate for this care setting. It is important for further work to be undertaken in order to validate such a tool internationally. Whilst all the participating sites were NHs undertaking GSFCH Programme this limitation is mitigated as the NHs had not actually implemented this programme. It is a two year programme and the questionnaires were sent during the first 15 months of its implementation. A final limitation was that its use within this study was in NHs in south-east England and thus the generalisability of findings to other care settings is unknown. Other factors may have resulted $\mathrm{n}$ experice eg culture leadership etc

\section{CONCLUSION:}

This study reports the experience of end-of-life care as perceived by bereaved relatives in UK NHs. The adapted FPCS used in this study was well received. Learning from and improving practice will be enabled if further validation of FPCS for care homes across countries occurs. NH organisations use satisfaction surveys for many aspects of care in order to evaluate the quality of care they provide but rarely specifically evaluate end-oflife care. Commissioners of care for frail older people might also be interested in such a tool. 
Acknowledgements - we would specifically like to thank Ruth Ward and Sandra Munday (volunteers on the Care Home Project Team at St Christopher's) for all their help and support in collating the data for the FPCS.

Key points for policy, practice, and/or research

- The quality of end of life care is rarely audited by care homes

- Bereaved families are a good proxy for end of life care in care homes

- Bereaved families are keen to be involved in evaluating end of life care in care homes

- Bereaved families would like more medical input to care homes to support the care of family members

- Recognising dying is fundamental to managing good care in the last weeks of life 


\section{REFERENCES:}

Alzheimers Society (2013) Low Expectations Attitudes on choice, care and community for people with dementia in care homes. London: Alzheimer's Society. http://www.alzheimers.org.uk/site/scripts/download info.php?fileID=1628 [Accessed 22 April 2017]

Baar F. (2002) 'The role of the physician in nursing home care in The Netherlands' in Hockley, J. and Clark, D. (eds.) Palliative Care for Older People in Care Homes. Buckingham: Open University Press.

BGS. (2011) Quest for Quality: Joint Working Party Inquiry into the Quality of Healthcare Support for Older People in Care Homes: a call for leadership, partnership and quality improvement. London: British Geriatric Society.

Brazil K. (2009) 'Assessing care needs and satisfaction with health care delivery' in. Hudson, P. and Payne, S. (eds.) Family Carers and Palliative Care. Oxford: Oxford University Press.

Brazil K, Krueger P, Bedard M, Kelley L, McAiney C, Justice C, et al. (2006) Quality of care for residents dying in Ontario long-term care facilities: findings from a survey of directors of care. Journal of Palliative Care, 22(1) pp. 18-25.

DH (2013) End of Life Care Strategy $-4^{\text {th }}$ Annual Report. London: Department of Health. Available from:

https://www.gov.uk/government/uploads/system/uploads/attachment_data/file/136486/En d-of-Life-Care-Strategy-Fourth-Annual-report-web-version-v2.pdf [Accessed 22 April 2017] 
Coleman J., Levy J., Wiggins S. and Kinley J. (2016) Using a new end-of-life care plan in nursing homes Nursing \& Residential Care 19(1), 38-41.

Ellershaw J \& Wilkinson S. (2003) Care of the dying: a pathway to excellence. Oxford: Oxford University Press.

Fisher R \& Ross M (2000) A guide to end-of-life care for seniors. Ottawa: University of Toronto. University of Ottawa.

Froggatt K, Vaughan S, Bernard C \& Wild D. (2009) Advance care planning in care homes for older people: an English perspective. Palliative Medicine, 23 pp. 332 - 8. GSF (2009) GSF Care Home Briefing paper. A framework to enable a gold standard of care for all people nearing the end of life. National GSF Centre: Walsall

Handley M, Goodman C, Froggatt K, Mathie E, Gage H, Manthorpe J, et al. (2014) Living and dying: responsibility for end-of-life care in care homes without on-site nursing provision - a prospective study. Health \& Social Care in the Community, 22(1) pp.22-29 doi: 10.1111/hsc.12055.

Hannah J, Durkin M \& McLackland D. (2005) Annual Report of Enhanced Services: nursing homes medical practice - report and recommendations. NHS Greater Glasgow: Greater Glasgow Primary Care NHS Trust. Available from: http://library.nhsggc.org.uk/mediaAssets/Hidden\%20Storage/nhsggc_care_homes_report recommendations_2005-12.pdf [Accessed 22 April 2017] Healthwatch (2017) What is it like to live in a care home? http://www.healthwatch.co.uk/sites/healthwatch.co.uk/files/20170815_whats_it_like to_1 
ive in a care home findings from the healthwatch network.pdf [Accessed 17th August 2017)

Hanson LC \& Henderson M. (2000) Care of the dying in long-term care settings. Clinics of Geriatric Medicine, 16(2) pp. 225-37.

Husebo B, Ballard C, Sandvik R, Nilsen O B \& Aarsland D. (2011) Efficacy of treating pain to reduce behavioural disturbances in residents of nursing homes with dementia: cluster randomised clinical trial. British Medical Journal, vol 343:d4065. doi: 10.1136/bmj.d4065.

Harrison J, McKay I, Grant P, Hannah J, Quinn T (2016) Appropriateness of unschedules hospital admissions from care homes. Clinical Medicine, 16(2): 103-8

Hockley J, Kinley J. and Stewart R (2013). The use of Action Learning Sets to enhance Facilitation of the Gold Standards Framework in Care Homes End-of-Life-Care Programme: the intervention arm of a Custer Randomised Control Trial. Journal of Palliative Care, 28(3) pp. 228.

Hockley J, Watson J, Oxenham D \& Murray S. (2010) The integrated implementation of two end-of-life care tools in nursing care homes in the UK: an in-depth evaluation. Palliative Medicine, 24(8) pp. 828-838.

Jacobs S. (2003) Addressing the problems associated with general practitioners workload in nursing and residential homes: findings from a qualitative study. British Journal of General Practice, 53, pp. 113-119.

Jeon Y, Fethney J \& Ludford I. (2012) Measuring Client Satisfaction in Residential Aged Care Settings: A Narrative Review of Instruments. The Internet Journal of Healthcare Administration 8(1). Available from: http://ispub.com/IJHCA/8/1/13747 [Accessed 22 April 2017]. 
Kaasalainen S, Brazil K, Akhtar-Danesh N, Coker E, Ploeg J, Donald F et al. (2012) The evaluation of an interdisciplinary pain protocol in long term care. Journal of the American Medical Directors Association, 13(7) p. 664.e1-664.e8.

Kinley J, Hockley J, Stone L, Dewey M, Hansford P, Stewart R, et al. (2013) The provision of care for residents dying in UK nursing care homes. Age and Ageing, 43(3) pp. $375-379$

Kinley J, Hockley J, Stone L, Levy J, Dewey M, Stewart R, et al. (2014) The effect of using high facilitation when implementing the Gold Standards Framework in Care Homes programme: a cluster randomised controlled trial. Palliative Medicine, 28(9) pp. 1099-1109.

Koloroutis M. (2004) Relationship-based care: a model for transforming practice. Minneapolis: Creative Healthcare Management.

Levy J, Conway F \& Kinley J (2016) Family Perception of Care Audit. London: St Christopher's Hospice \& Burdett Trust for Nursing. Available from: http://www.btfn.org.uk/library/directory_listings/341/Family\%20Perception\%20of\%20C are\%20Audit\%20Project\%20Final\%20Report\%20June\%202016.pdf [Accessed 4 May 2017].

Li Q, Zheng N T \& Temkin-Greener H (2013). Quality of end-of-life care of long-term nursing home residents with and without dementia. Journal of American Geriatric Society, 61(7) pp. 1066-73. 
Lunney J R, Lynn J, Foley D, Lipson S \& Guranlik J. (2003) Patterns of Functional Decline at the End of Life. JAMA, 289(18) pp. 2387-92.

Mezey M, Mitty E \& Burger S. (2009) Nursing Homes as a clinical site for training geriatric health care professionals. Journal of the American Medical Directors, 10, pp. 196-203.

Murray S, Kendall M, Boyd K \& Sheikh A. (2005) Illness trajectories in palliative care. British Medical Journal, 330, pp. 1007-1011.

NHS England (2016) The framework for enhanced health in care homes https://www.england.nhs.uk/wp-content/uploads/2016/09/ehch-framework-v2.pdf [Accessed 17th August 2017]

NHS England (2017) Next Steps on the NHS Five Year Forward View https://www.england.nhs.uk/wp-content/uploads/2017/03/NEXT-STEPS-ON-THE-NHSFIVE-YEAR-FORWARD-VIEW.pdf [Accessed 17th August 2017]

Oliver D P, Porock D \& Zweig S. (2004) End-of-life care in U.S. nursing homes: a review of the evidence. Journal of American Medical Directors Association, 5(3) pp. 147-55.

Parker D \& Hodgkinson B. (2010) A comparison of palliative care outcome measures used to assess the quality of palliative care provided in long-term care facilities: a systematic review. Palliative Medicine, 25(1) pp. 5-20.

Seers K, Cox K, Crichton N, Edwards R, Eldh A, Harvey G et al. (2012) FIRE (Facilitating Implementation of Research Evidence): a study protocol. Implementation Science, 7(25) http://www.implementationscience.com/content/7/1/25 [Accessed 22 April 2017]. 
Stone L., Kinley J. and Hockley J. (2013) Advance care planning in care homes: the experience of staff, residents and family members. International Journal of Palliative Nursing 19(11), 550-557.

Swagerty D (2017) Integration of Palliative Care in U.S. Nursing Homes.

AGG/WHO/SFGG Workshop - June 4th \& $5^{\text {th }} ; 2$ 2010. Toulouse, France. Available from: http://www.iagg.info/data/SWAGERTY_1_Integrating_Palliative_Care_in_US_Nursing Homes.pdf [Accessed 22 April 2017].

Travis SS, Loving G, McClanahan L \& Bernard M. (2001) Hospitalization patterns and palliation in the last year of life among residents in long-term care. Gerontologist, 41(2) pp. 153-60.

Vivat et al (2017) The international phase 4 validation study of the EORTC QLQSWB32: A stand-alone measure of spiritual well-being for people receiving palliative care for cancer. European Journal of Cancer Care https://doi.org/10.1111/ecc.12697 (Accessed 17th August 2017).

Vohra J, Brazil K \& Szala-Meneok K. (2006) The last word: family members descriptions of end of life care in long term care facilities. Journal of Palliative Care, 22(1) pp. 33-39.

Wilkinson A. \& Lynn J. (2005) Caregiving for advanced chronic illness patients. Techniques in Regional Anesthesia and Pain Management, 9, pp. 122-132. Wordsworth H. (2014) Health ministry through local faith communities: a European perspective. Community Practitioner, 87(1) pp. 24-27. 
Zheng NT, Temkin-Greener H (2010) End-of-Life Care in Nursing Homes: The Importance of CNA Staff Communication. Journal of the American Medical Directors Association, 11(7), pp.494-499 
Table 1: Characteristics of those returning the FPCS

\begin{tabular}{|l|l|}
\hline & $\mathbf{n ~ ( \% )}$ \\
\hline Bereaved Relatives* (n=366) & \\
\hline Gender (\% female) & $242(66.1)$ \\
\hline Age (years) & \\
\hline $30-49$ & $28(7.9)$ \\
\hline $50-59$ & $117(33.1)$ \\
\hline $60-69$ & $122(34.6)$ \\
\hline $70-79$ & $49(13.9)$ \\
\hline $80+$ & $36(10.2)$ \\
\hline Relationship to resident & \\
\hline Son / daughter & $218(60.0)$ \\
\hline Spouse / partner & $67(18.5)$ \\
\hline Sibling & $14(3.8)$ \\
\hline Friend & $10(2.8)$ \\
\hline Son-in-law / daughter-in-law & $8(2.2)$ \\
\hline Parent & $1(0.3)$ \\
\hline Other & $45(12.4)$ \\
\hline
\end{tabular}

*Denominators vary among variables due to missing responses 
Table 2: Nursing Care Home (NH) characteristics

\begin{tabular}{|l|l|}
\hline \multicolumn{1}{|c|}{ NHs(n=37) } & \multicolumn{1}{|c|}{$\mathbf{n}(\mathbf{\%})$} \\
\hline For profit & $26(70.3)$ \\
\hline Part of a large cooperative & $20(54.1)$ \\
\hline General Practitioner (GP) & \\
practices attending NH (n) & \\
1 & $16(43.2)$ \\
2 & $8(21.6)$ \\
\cline { 2 - 2 } 3 & $5(13.5)$ \\
\cline { 2 - 2 } 5 & $3(8.1)$ \\
\cline { 2 - 2 } 6 & $2(5.4)$ \\
\cline { 2 - 2 } 6 & $3(8.1)$ \\
\hline
\end{tabular}


Table 3: Family Perception of Care Scale (adapted)

\begin{tabular}{|l|l|l|}
\hline \multicolumn{1}{|c|}{ Item } & \multicolumn{1}{|c|}{ n } & \multicolumn{1}{|c|}{ mean (SD) } \\
\hline The staff were friendly to me & 361 & $6.5(1.0)$ \\
\hline The staff treated my family member with dignity & 360 & $6.2(1.2)$ \\
\hline The staff spent enough time with my family member & 352 & $5.7(1.5)$ \\
\hline The staff provided comfort to my family member & 359 & $5.9(1.4)$ \\
\hline The staff were sensitive to the needs of my family member & 358 & $5.9(1.4)$ \\
\hline The staff kept me informed about my family member's health & 359 & $5.9(1.5)$ \\
\hline The staff kept me updated based on what I wanted to know & 359 & $5.9(1.5)$ \\
\hline The staff spoke to me in a way that was easy to grasp & 355 & $6.2(1.1)$ \\
\hline $\begin{array}{l}\text { The staff described what to expect as my family member came } \\
\text { closer to death }\end{array}$ & 297 & $5.3(1.9)$ \\
\hline $\begin{array}{l}\text { The staff informed me about care options during my family } \\
\text { member's last days }\end{array}$ & 279 & $5.2(1.9)$ \\
\hline The staff involved me in the planning of care & 319 & $5.4(1.7)$ \\
\hline $\begin{array}{l}\text { There was a plan of care tailored specifically to the needs of my } \\
\text { family member }\end{array}$ & 326 & $5.6(1.6)$ \\
\hline $\begin{array}{l}\text { The staff took note of an action I thought necessary and we } \\
\text { worked things out appropriately }\end{array}$ & 295 & $5.6(1.6)$ \\
\hline $\begin{array}{l}\text { My family member's pain was eased to the greatest extent } \\
\text { possible }\end{array}$ & 321 & $5.9(1.4)$ \\
\hline Other symptoms were eased to the greatest extent possible & 317 & $5.8(1.5)$ \\
\hline $\begin{array}{l}\text { The staff informed me when they thought that my family member } \\
\text { was dying }\end{array}$ & 297 & $5.6(1.9)$ \\
\hline The staff welcomed me to stay with my family member & 308 & $6.0(1.5)$ \\
\hline $\begin{array}{l}\text { The staff helped me to be involved in the care of my family } \\
\text { member }\end{array}$ & 318 & $5.7(1.6)$ \\
\hline My family member's room offered privacy & 349 & $6.4(1.0)$ \\
\hline Spiritual support was at hand for my family member & $5.2(1.7)$ \\
\hline $\begin{array}{l}\text { The staff asked about and met important rites and rituals of my } \\
\text { family member / us }\end{array}$ & 248 & $5.0(1.7)$ \\
\hline There was someone there for my family member to talk to & 316 & $5.7(1.6)$ \\
\hline There was enough staff to deal with my concerns & 338 & $5.6(1.7)$ \\
\hline $\begin{array}{l}\text { Overall, I am satisfied with the end-of-life care that was given to } \\
\text { my family member }\end{array}$ & 340 & $5.9(1.6)$ \\
\hline
\end{tabular}


Table 4: Items from FPCS that bereaved relatives identified as priorities for excellent care

\begin{tabular}{|lc|}
\hline Item & n \\
\hline The staff treated my family member with dignity & $163(50.8)$ \\
\hline My family member's pain was eased to the greatest extent possible & $119(37.1)$ \\
\hline The staff were sensitive to the needs of my family member & $94(29.3)$ \\
\hline The staff provided comfort to my family member & $5316.50)$ \\
\hline Other symptoms were eased to the greatest extent possible & $52(16.2)$ \\
\hline
\end{tabular}

